\title{
Accelerate Innovation by Using Cloud Computing Technology
}

\author{
Kaninda Musumbu \\ LaBRI-Université de Bordeaux 351, Cours de la \\ Libération \\ Talence, France \\ email: musumbu@labri.fr
}

\author{
Awa Yaram Diop \\ Cloud Specialist Immeuble Atrium Center \\ Talence, France \\ email: yaramdiop@gmail.com
}

\begin{abstract}
An information system can be seen as a set of digital and human resources organized to handle, spread and store information. Various standards, models of evaluation and methods of analysis of the risks exist to arrest the information system security. This one can be approached by a technological point of view, certainly, but also by a human point of view. The question of the knowledge carried by the people, as well as the confidence which they give to the system and which the system gives to them are crucial to assure the information system security in organizations. The Cloud Computing Technology offers many and divers applications, services and infrastructure to realize your ideas. Your team can now be more productive, collaborative and innovative, with more flexibility. Ideas can thus be carried out faster in the market. But the problem of security stay open.
\end{abstract}

Keywords- information system; cloud computing technology; security.

\section{INTRODUCTION}

Without the right tools, an idea may never emerge. The Cloud Computing Technology offers many and divers applications, services and infrastructure to realize your ideas. Your team can now be more productive, collaborative and innovative, with more flexibility. Ideas can thus be carried out faster in the market. The cloud has become one way end-users are seeking to empower themselves The next generation of Middleware should provide new abstractions for better programmability, performance, scalability and security, as well as for the integration of essential features such as elasticity and green awareness, Cloud computing is access computer resources that are somewhere through internet. It can be accessed free of charge, as is the case with the webmail. Or subscription, with a guaranteed level of service. Companies are buying and building, and some are charged as are water, gas or electricity: you pay for consumption. As the electric current, one can consume as much as one wants. Virtually power is infinite. Cloud computing is an economic revolution but not technological one. Because it is based on long-established technology. Crossover operation and self-adaptation of mutation rates provides a wide selection of models and choices. Choices include automatic adaptation of operator probabilities and a dynamic ranking mechanism, etc.

What is the advantage of cloud computing? It is especially economical. If you are a very small company, this will launch a service with no capital investment in hardware. Thus, virtually no start-up of the software is investing in heavy equipment, today. The second advantage is to benefit from economies of scale that have economic repercussions. For example, the computing resources that

are not used by French companies night, are by companies on the other side of the planet.

It's like a plant that would turn round the clock, and whose resources are shared. This allows for example to companies such as e-tailers that have a Christmas peak load and less load the rest of the year, to have the necessary resources during this peak capacity without investing in them would not use the rest of the year. That's how Amazon has launched cloud computing services from. They had invested in huge capacity and sought to dampen.

The safety data was a big hurdle for cloud computing adoption, now we can say that this problem is largely solved. For more security for companies, there are private clouds, which are not shared. The goal is to have the same benefits, but to keep control of his environment. This type of service is offered by many actors such as private hosts called Application Service Provider ( ASP).

The main purpose of our paper is to show that the service company that sells some cloud technology does not actually cross the cloud, it has big base and provides powerful server and simulates cloud technology. the use and customers must be informed and awaken their distrust "vis-à-vis" these practice,

The paper is divided in two parts. In section 2 we present cloud computing technology and section 3 is devoted to illustrate different possibilities to trick the user. Finally, we conclude by presenting some experimental results to illustrate its effectiveness.

\section{CLOUD COMPUTING}

\section{A. Motivation}

The cloud allows to outsource computing power, storage capacity. Can you imagine, in the future, no longer need to buy ever more powerful computers and hard drives to work and store data?

Not really. Because he always needs a terminal to present the data with a level of ergonomics increasingly developed: augmented reality, 3D, etc. Many treatments are performed in the cloud, but the growing sophistication of interfaces requires power locally. In addition, contextual computing, in future, require that the computer knows in which situation the user is located. We will need to interact 
with systems more intelligent. The cloud will increase the capacity of its workstation.

These applications traditionally have required a conservative approach to maintenance. As mission - critical applications, they need a rock solid foundation, high availability mechanisms, and a light touch when doing updates, which must be painstakingly planned. The goal is to reduce risk to the business by ensuring that crucial applications are always on. The need to minimize risk to these applications is one of the reasons some IT departments have reputations of being stodgy

and unyielding. In fact, the IT group is simply trying to keep the business running. Change makes that a difficult charge.

With a cloud application, simply open your browser, log in and get to work.

For your business remotely, a CRM cloud provides access to all the necessary information from a mobile terminal. It is also possible to update in real time the contact data. No more waiting for his return to the office, your data is always fresh and comprehensive. In addition, your business managers know exactly which ones will be signed and when, from the terminal of their choice.

And all this without hardware to purchase and manage, no software to install and update: these are entirely the responsibility of the cloud company that runs your application.

\section{B. Public Cloud}

A cloud is called a "public cloud" when the services are rendered over a network that is

Open for public use. Public cloud services may be free. Technically there may be little or no difference between public and private cloud architecture, however, security consideration may be substantially different for services (applications, storage, and other resources) that are made available by a service provider for a public audience and when communication is effected over a non-trusted network. Generally, public cloud service providers like Amazon Web Services (AWS), Microsoft and Google own and operate the infrastructure at their data center and access is generally via the Internet. AWS and Microsoft also offer direct connect services called "AWS Direct Connect" and "Azure ExpressRoute" respectively, such connections require customers to purchase or lease a private connection to a peering point offered by the cloud provider.

\section{Private Cloud}

Private cloud is cloud infrastructure operated solely for a single organization, whether managed internally or by a third-party, and hosted either internally or externally.[2] Undertaking a private cloud project requires a significant level and degree of engagement to virtualize the business environment, and requires the organization to reevaluate decisions about existing resources. When done right, it can improve business, but every step in the project raises security issues that must be addressed to prevent serious vulnerabilities. Self-run data centers[87] are generally capital intensive. They have a significant physical footprint, requiring allocations of space, hardware, and environmental controls. These assets have to be refreshed periodically, resulting in additional capital expenditures.

\section{MANAGEMENT OF DATA SECURITY}

Cloud brings an optimal safety (security) which the traditional and internal infrastructures of a company do not have. The data are automatically protected and protected by security systems of the company. This means as well as in case of loss or of flight, all your data are not lost, nor accessible by a third person, these being stored in the computer but in the "cloud".This is the idealistic vision but when is - it in the real world.

\section{A. Cloud Computing Security}

That is an evolving sub-domain of computer security, network security and information security.

It refers to a broad set of policies, technologies, and controls deployed to protect data, applications, and the associated infrastructure of cloud computing.

\section{B. Data Confidentiality}

Data confidentiality is the property that data contents are not made available or disclosed to illegal users. Outsourced data is stored in a cloud and out of the owners' direct control. Only authorized users can access the sensitive data while others, including CSPs, should not gain any information of the data. Meanwhile, data owners expect to fully utilize cloud data services, e.g., data search, data computation, and data sharing, without the leakage of the data contents to CSPs or other adversaries.

\section{Data Access Controllability}

Access controllability means that a data owner can perform the selective restriction of access to his data outsourced to cloud. Legal users can be authorized by the owner to access the data, while others can not access it without permissions. Further, it is desirable to enforce finegrained access control to the outsourced data, i.e., different users should be granted different access privileges with regard to different data pieces. The access authorization must be controlled only by the owner in untrusted cloud environments. services to the cloud.

\section{Data Integrity}

Data integrity demands maintaining and assuring the accuracy and completeness of data. A data owner always expects that his data in a cloud can be stored correctly and trustworthily. It means that the data should not be illegally tampered, improperly modified, deliberately deleted, or maliciously fabricated. If any undesirable operations corrupt or delete the data, the owner should be able to detect the corruption or loss. Further, when a portion of the outsourced data is corrupted or lost, it can still be retrieved by the data users. Location of the data constitute a mattering aspect folding the security of data, indeed according to the laws of the country, the integrity of data can be guaranteed more at least, it belongs to the customer to know 
how to judge the importance of its data and costs which it is ready to pay for their integrity.

\section{LEVERS OF ADOPTION OF THE CLOUD}

In the current economic environment, the capacity to be answered the evolutionary needs for the customers establishes(constitutes) a key competitive advantage. Things being what they are, companies look constantly for ways(means) to improve their suppleness to adapt itself to the demands(requests) of the market. A third(third party) of the persons in charge questioned by the study led by THE ECONOMIST think that Cloud can help in this sense(direction) and quote among its main advantages the adaptability to the market. By allowing the companies to adjust quickly their processes and to adapt their products and their services(departments) to answer the changeable needs for the market, Cloud facilitates the innovation and the fast prototype, and helps companies to enter more quickly on the market.

The flexibility is one of the main reasons which urged a lot of companies to adopt Cloud. The major advantage of the cloud is its capacity to reduce the fixed computing costs and to pass, a cost structure based on a payment in the more variable use(custom). Cloud can help one Organization to reduce its costs to operating expenses. The investments in computing, which include generally the software licenses, the servers and the network equipments, be more expensive and more difficult to plan that operating with the applications in Cloud.

\section{A. An Important Point of Vigilance}

The availability of the service is a mattering element on which one the customer has to make sure to have a guarantee of service, The customer has to know the ceiling of availability required for his workloads various which he integrates into the cloud and to define the SLAs, with the supplier, which cover this availability. The customer has to make sure, that a servic of support is supplied with the cloud service (department).

\section{CONCLUSION}

The main objective of this article is to bring to the foreground the importance of the information system security and its complexity of a not only technological point of view, but also human, by taking advantage of the practice and the various areas of research in the context of Cloud computing.

In short, the cloud is a solution for small businesses, which often lack the resources to invest in a highly secure computer equipment. That said, large companies can also benefit by exporting some of their services to the cloud.

But in any case, we are more escapes, the cloud has become ubiquitous in our society and

Hybrid cloud is a composition of two or more clouds (private or public) that remain distinct entities but are bound together, offering the benefits of multiple deployment models. Hybrid cloud can also mean the ability to connect collocation, managed and/or dedicated services with cloud resources with more security guaranteed.

\section{REFERENCES}

[1] Bégin, M., An egee comparative study: Grids and cloudsevolution or revolution, in Enabling Grids for E-Science. 2008, CERN.

[2] L. Vaquero, L. Rodero-Merino, J. Caceres and M. Linder. A Break in the Clouds: Towards a Cloud Definition, ACM SIGCOMM Computer Communication Review 39(1), 50 - 55, January 2009.

[3] Hugos, M., \& Hulitzky, D. (2011). Business in the cloud: What every business needs to know about cloud computing. Hoboken, NJ John Wiley \& Son, Inc..

[4] Kushida, K., Murray, J., \& Zysman, J. (2011). Diffusing the cloud: Cloud computing and implications for public policy. Journal of Industry, Competition and Trade, 11(3), 209-237.

[5] Mather, T., Kumaraswamy, S., \& Latif, S. (2009). Cloud security and privacy. Sebastopol, CA: O'Reilly Media, Inc..

[6] Rosenberg, J., \& Mateos, A. (2011). The cloud at your service: The when, how, and why enterprise cloud computing. Greenwich, CT: Manning Publications Co. 